\title{
Out, out, brief candle?
}

Time is slowly running out for life on Earth.

\section{Impossible Extinction: Natural \\ Catastrophes and the Supremacy of the Microbial World \\ by Charles C. Cockell \\ Cambridge University Press: 2003. 192 pp. \\ $\mathfrak{E} 18.95, \$ 28$ \\ The Life and Death of Planet \\ Earth: How the New Science of \\ Astrobiology Charts the Ultimate \\ Fate of our World \\ by Peter D. Ward \& Donald Brownlee \\ Piatkus Books/Time Books: 2003. 256 pp. \\ $£ 16.99 / \$ 25$}

\section{Norman H. Sleep}

One of the great intellectual achievements of the past millennium was realizing that the Sun is a star and the Earth is a planet. Over 400 years, this idea has gone from an excuse to burn the astronomer Giordano Bruno at the stake to a banality reserved for trick questions such as: "What is the nearest star to the Earth?" Reaction to the rank odour of biblical catastrophism and creationism has prejudiced serious consideration of extraterrestrial affairs in geology and biology. This has now changed, the main wake-up call being the discovery that an asteroid impact wiped out a vast number of species, including the dinosaurs, 65 million years ago.

A chief beneficiary of this change is astrobiology, the science of planetary habitability, including the effects of extraterrestrial processes on the evolution of life on Earth, which has become a hot topic. Charles Cockell, Peter Ward and Donald Brownlee add to the list of popular books on this subject. Both books focus on global sterilization and the finite tenure of life on a habitable planet, but Cockell concentrates on microbes, whereas Ward and Brownlee look at animals.

These treatments bring different emphases: that it is quite hard to sterilize all the microbes on a planet, and that the days of animals are numbered by a few hundred million years. But the authors agree on the salient conclusions of astrophysics, geology and biology. The Solar System formed about 4.5 billion years ago. Microbes inhabited Earth by the time of the first good geological record, 3.5-3.8 billion years ago, perhaps even earlier. Our Sun's useful life is limited: its luminosity has already increased by $30 \%$, and will continue to increase, until, 7 billion years from now, the red-giant stage will finally incinerate the Earth. In addition, the tectonic processes that continually renew the Earth's surface are on the wane.

I found Ward and Brownlee's book the easier to read. Its organization around the



Flaming Dune: as the Sun heats up, the Earth could become increasingly hot, dry and inhospitable.

various vicissitudes that await life works well, using an analogy to the progressive failure of various organ systems of an aged person. We can expect that as the Sun waxes, the weathering of rocks will reduce atmospheric $\mathrm{CO}_{2}$ to low levels, shutting down plant life and leaving little food or oxygen for animals. Still later, the greenhouse will become hotter, ending with either the loss of the ocean's hydrogen to space or a runaway period of oven-like conditions at the surface. The authors correctly note that the former possibility leaves us with Dune - a hot, dry planet that is still habitable by microbes, and perhaps by animals near the poles - whereas the latter leaves the surface sterile. In the meantime, the continents may group together, fouling the global climate, or plate tectonics may shut down, leaving us, as in the film Waterworld, with a water-covered planet from which all topography has been eroded.

In contrast, I found the organization of Cockell's book - on a trip of the Solar System once around the galaxy — rather contrived. As he admits, galactic effects on the Earth have been, and probably will remain, small. Any effect has more to do with our position relative to the galactic plane and to the spiral arms with giant molecular clouds and young stars than to absolute position relative to distant galaxies. This quibble aside, the book is worth reading. A highlight is the discussion of why we should not generalize too much from the actual state of the Earth. Cockell focuses on the presence of our large Moon. I might add that intelligent deep-sea organisms could probably come up with many reasons why intelligence could not arise on land, and that organisms circling a star ejected from its galaxy might think the plane of the galaxy to be an unattractive place.

Both books have the obligatory and entertaining discussion about how a hightech civilization could avoid or at least delay its doom. We all like to feel that our distant descendants, or at least our more distant microbial relatives, will survive. It is easy to come up with suggestions to be filed away for 100 million years. Importantly, both books show that the Earth is not a truly safe place even now. There are hazards that we can do something about: global warming by the burning of fossil fuels; the more distant threat of advancing glaciers; and the everpresent danger of impacts from asteroids and comets. Science provides both the technology to cause trouble for ourselves and the knowledge to foresee and avoid that trouble.

Astrobiology derives from several fields that until recently had little interaction. An inevitable result is that it is difficult for one or even two authors to cover fully this rapidly evolving field. Specialists will quibble with various details and prefer more treatment of their own fields; my own include the effects of global tectonics, geochemical cycles and large asteroid impacts on early life. More 
bothersome is the fact that both books contain some errors, so care is needed in using them as secondary sources. For example, Ward and Brownlee give the formation rate of continents as 650-1,300 cubic kilometres per year, a factor of about 1,000 too high. And it seems unlikely that global biomass drops by several thousandfold in an ice age. In the other book, Cockell's Earth has a mainly iron mantle, and his surface of Venus is younger than 250 million years and still active, rather than all about 500 million years old.

Having said that, I recommend both books to the lay reader. They can also be quickly read by a professional astrobiologist and do provide insight. And they would serve as supplemental reading (but not as primary texts) for a non-major class on planetary habitability or astrobiology.

Norman H. Sleep is in the Department of

Geophysics, Stanford University, Stanford, California 94305, USA.

\section{A stratospheric}

\section{success}

\section{Protecting the Ozone Layer:}

by Edward A. Parson

Oxford University Press: 2003. 396 pp. \$65

\section{Protecting the Ozone Layer:}

The United Nations History

by S. O. Anderson and K. M. Sarma

Earthscan: 2002. 544 pp. \$65, £40

\section{Charles Herrick}

When a scientific finding emerges from the field or laboratory, it undergoes a profound transition, changing from a well-formulated, discipline-bound construct into a dynamic and perhaps unruly component of a complex and interactive social system. The societal value of science and technology is created at the interface between laboratory and society, residing in neither alone, to paraphrase my friend Daniel Sarewitz. There is no direct and necessary linkage between the structure of physical reality and the manner in which society applies its scientific understanding of that structure. Rather, the social application of scientific knowledge and technological achievement is mediated through the formation and execution of policies.

Two new books, both entitled Protecting the Ozone Layer, chronicle the stratospheric phenomenon of ozone depletion as a window to explore the complex and dynamic relationships between science, technology and public policy. Although no small chore, as they have a combined length of almost 1,000 pages, I encourage readers to tackle both books, as their complementarity is fortuitous and sometimes quite illuminating.

Drawing on such fields as international-
Science and Strategy relations theory and political sociology, Edward Parson outlines the formulation and implementation of the Montreal Protocol on ozone-depleting substances and its subsequent annexes, in terms of constructs such as epistemic communities and transnational issue networks. This provides the theoretical basis for his account of how scientific assessments, as distinct from scientific results per se, contribute to the development and maturation of adaptive policy regimes. Anderson and Sarma, on the other hand, offer a historical account based primarily on documentation and the recollections of key

Parson weaves a convincing account of the dynamic conditions that caused the initial slow progression of ozone negotiations between 1978 and 1986, and that then underpinned the swift consolidation of the Montreal Protocol by 1988. Briefly, he argues that scientific findings, considered alone, have little influence on the process of policy formulation. Rather, scientific results acquire credibility, salience and legitimacy only when compiled and interpreted in the context of a formalized, multi-party assessment process. As he writes, assessments "make key scientific statements and their implications common knowledge among policy actors" in the sense "that all parties know them, all know that all know them, and so on". In this way, assessments serve to bound and delimit the domain of reasonable negotiation and, once established, hasten regime formation.

Parson also argues that the formalized institutional impetus to move negotiations forward. This was so effective that Mostafa Tolba, then director of the United Nations Environment Programme (UNEP), was able to "browbeat" recalcitrant delegations into agreeing with provisions that were to become the Montreal Protocol.

Anderson and Sarma put forward a different view: that it was Tolba's truly exceptional leadership qualities that clinched the negotiations. Indeed, Sarma and Anderson's book is full of wonderful vignettes that demonstrate the creativity and dedication of the government scientists and civil servants who worked for over a decade to create a workable global environmental-

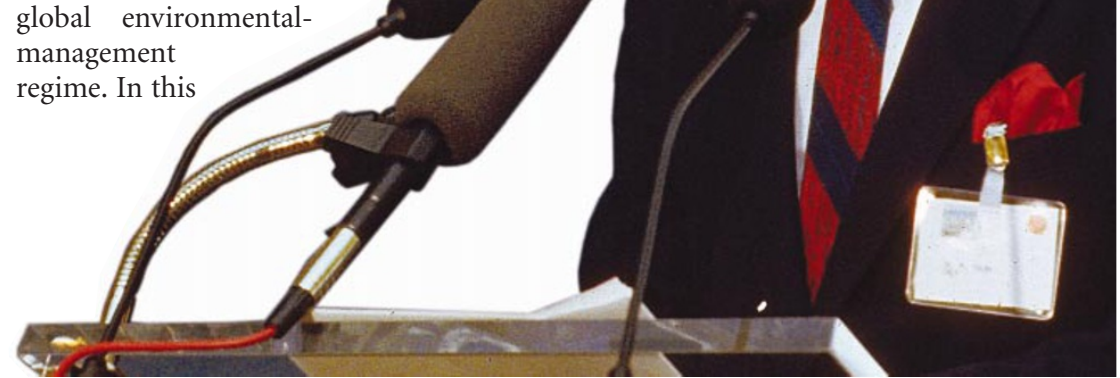

Tough talking: effective scientific backing helped Mostafa Tolba forge the basis of the Montreal Protocol.

context, many of the individuals quoted or paraphrased cite the importance of informal groups and of a strong and inclusive consensus process that tended to override legalistic hair-splitting. So, did the institutional setting empower the actors, or did an exceptional group of actors combine to stretch the bounds of game-theoretic interpretation?

Both books do a good job of describing the evolution of stratospheric science and its relation to ozone depletion, chronicling the accomplishments of Sidney Chapman, G. M. B. Dobson, Lester Machta, Mario Molina and Sherwood Rowland, along with other pioneers of atmospheric science. They also describe how various stakeholder communities drew from this science base to support their positions and negotiations. I found myself wondering whether history would have been significantly different if industry had chosen an alternative field of battle and engaged the ozone-depletion debate from the perspective of cancer epidemiology, rather than atmospheric chemistry. Epidemiological studies show a rising incidence of melanoma, but evidence linking these skin cancers to increasing ultraviolet radiation, as the ozone layer was depleted, is complex and equivocal, and might have made a far less compelling case.

Neither book focuses heavily on the predicted and/or postulated effects of ozone depletion, merely noting, as Parson put it, that the policy debate was "pervaded by a general sense that effects did not matter". players in the ozone-treaty process. assessment structure resulted in a powerful

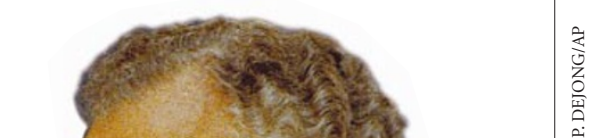

